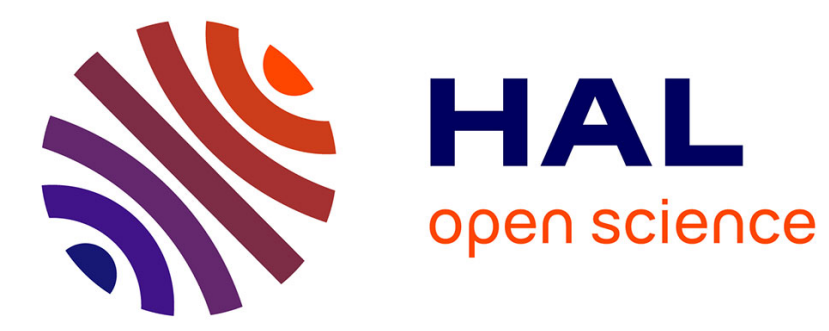

\title{
César orateur. Cassius Dion, Histoire romaine, 43.15-18 Antoine Jayat
}

\section{To cite this version:}

Antoine Jayat. César orateur. Cassius Dion, Histoire romaine, 43.15-18. Veni, Vidi, Scripsi: écrire l'histoire dans l'Antiquité, UN@ Éditions; UN@ Éditions, pp.49-62, 2021, 978-2-35613-381-6. 10.46608/primaluna7.9782356133793.3 . halshs-03151031

\section{HAL Id: halshs-03151031 https://shs.hal.science/halshs-03151031}

Submitted on 9 Mar 2021

HAL is a multi-disciplinary open access archive for the deposit and dissemination of scientific research documents, whether they are published or not. The documents may come from teaching and research institutions in France or abroad, or from public or private research centers.
L'archive ouverte pluridisciplinaire HAL, est destinée au dépôt et à la diffusion de documents scientifiques de niveau recherche, publiés ou non, émanant des établissements d'enseignement et de recherche français ou étrangers, des laboratoires publics ou privés. 


\title{
CÉSAR ORATEUR \\ CASSIUS DION, HISTOIRE ROMAINE, 43.15-18
}

\author{
Antoine Jayat
}

Après sa victoire, à Thapsus (6 avril 46 a.C.), sur les forces pompéiennes en Afrique, C. Iulius Caesar marque une étape supplémentaire dans son statut de vainqueur des guerres civiles, qui sera parachevé avec la victoire de Munda l'année suivante (17 mars 45 a.C.).C'est après Thapsus que l'on place traditionnellement le début d'une période que l'on a longtemps appelée la "monarchie césarienne", car le pouvoir de César est désormais sans partage".

À Rome, fort du souvenir de Sylla, on peut craindre alors que le dictateur n'abuse de sa prééminence désormais totale - c'est du moins ce que décrit Cassius Dion, qui dépeint une Ville dans l'expectative et la crainte du comportement à venir de César, contraignant le dictateur à prendre la parole pour dissiper les craintes ${ }^{2}$. Prendre la parole au Sénat apparaît tout à fait logique pour un homme politique de cette envergure, et dont les sources littéraires conservées tendent de manière unanime à le qualifier d'orateur prolixe $\mathrm{d}^{3}$ et brillant ${ }^{4}$.

Or ce discours de César au Sénat par Cassius Dion est exceptionnel car unique dans nos sources, Cassius Dion étant le seul à avoir composé un discours de César au Sénat au lendemain de Thapsus ${ }^{5}$. Cela pousse F. Millar à envisager que ce discours n'a jamais été tenu et qu'il est une pure invention de $\operatorname{Dion}^{6}$, ce qui est tout à fait probable. R. Étienne suit le raisonnement de F. Millar et va jusqu'à voir dans ce discours composé par Dion un lieu stratégique où se

1 L'expression "monarchie césarienne" vient du titre de Meyer 1918, mais déjà Mommsen [1882-1886] 1992, 68-91 faisait remonter cette période au lendemain de Thapsus; récemment encore, Jehne 2008, 101-114 situait également le début de la "monarchie césarienne" à ce moment-là. Il nous semble cependant qu'aux yeux de Dion du moins, cette expression ne soit pas adaptée : nous développons cette idée dans notre travail de thèse en cours (Édition avec traduction et commentaire du livre 43 de I'Histoire romaine de Cassius Dion, sous la direction de V. Fromentin et d'E. Bertrand). Toutes les traductions des passages issus du livre 43 sont extraites de ce travail en cours. Les traductions du livres 44 sont également les nôtres. Pour les autres livres, nous nous appuyons, sauf mention contraire, sur la traduction de la CUF.

2 C.D. $43.15 .1-2$

3 Il ne reste cependant que quatorze titres de discours de César, répertoriés dans Malcovati 1955, 383397.

4 Dans le Brutus (Cic., Brut., 252 et 261), Atticus souligne l'elegentia des discours de César et l'usage des oratoria ornamenta. On trouve des qualificatifs semblables chez Salluste (Cat., 54.1), Quintilien (Inst., 10.1.114), Tacite (Dial. Orat., 21.5-6 et 25.3; Ann., 13.3), Pline le Jeune (Ep., 1.20.4), Suétone (Caes., 21.5 et 25.3), Plutarque (Caes., 3.2 et 7.8 ; Cat. Mi., 22.5), Fronton (Ad Ver., 2.10), Aulu-Gelle (NA, 19.8.3), Apulée (Apol., 95.5) et Appien (BC, 2.10). Voir Rambaud 1987.

5 Plutarque (Caes., 55.1) mentionne simplement que César adresse un discours au peuple, et dont

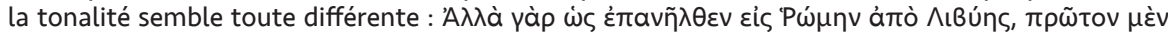

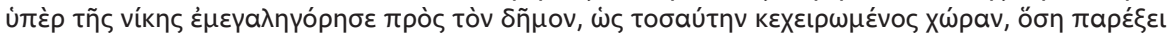

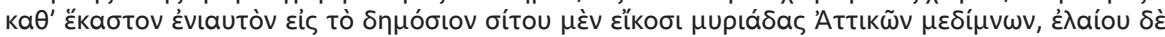

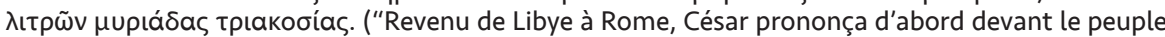
un discours où il exaltait sa victoire, disant notamment que les pays conquis étaient assez grands pour fournir chaque année à l'État deux cent mille médimnes attiques de blé et trois millions de livres d'huile".).

6 Millar 1964, 80-81. Burden-Strevens 2020, 174-175. 
donne à lire la manière dont Cassius Dion, historien de l'époque des Sévères, conçoit le pouvoir idéal ${ }^{7}$; nous serons amenés à revenir sur cette réflexion. Plus récemment, $M$. Coudry a montré comment le vocabulaire politique présent dans ce discours participe à caractériser le pouvoir césarien dans l'Histoire romaine ${ }^{8}$. C. Meier, enfin, semble considérer que ce discours a réellement eu lieu mais ne s'appuie sur aucun élément concret et ne cite par ailleurs aucune source ${ }^{9}$.

Mais ce discours constitue en réalité l'un des seuls textes, dans les sources historiques, à représenter César en orateur politique. Car si l'on met de côté les discours de César chez César ${ }^{10}$ et les discours de César que les sources présentent comme adressés à ses soldats ${ }^{11}$ - les contiones -, force est de constater que, dans les sources dont nous disposons, peu de textes donnent la parole à César pour lui faire incarner la figure de l'orateur politique. Un seul discours, ample et au style direct, nous semble comparable à celui que compose Dion au livre 43 de l'Histoire romaine : celui que compose Salluste à l'occasion du châtiment à infliger aux conjurés réunis derrière Catilina ${ }^{12}$. C'est d'ailleurs à propos du même sujet que certains discours sont rapportés chez Plutarque, mais brièvement et au style indirect $\mathrm{t}^{13}$ ou incluant parfois quelques mots de citation ${ }^{14}$. En dehors de ces textes, on trouve également quelques brèves évocations, au style indirect ou narrativisé, de discours de César au Sénat chez Appien ${ }^{15}$ et Cassius Dion ${ }^{16}$; ce dernier discours, qui est censé être tenu en 49 a.C., au moment où César occupe Rome après la fuite de Pompée au début de la guerre civile, sera également analysé car il éclaire celui de 46 a.C. de manière très importante.

César incarne de nombreuses figures différentes dans les sources littéraires : chef militaire, conquérant - Plutarque met en parallèle sa vie avec celle d'Alexandre -, chef politique, législateur... Quant à la figure de l'orateur, il l'incarne en réalité très peu - ou plutôt il l'incarne en trompe-l'œil, puisque les discours de César présents dans les sources sont abondants mais sont presque toujours des discours militaires et développent finalement bien davantage la figure du chef de guerre que celle de l'orateur ${ }^{17}$.

Étienne 1997, 235-239.

8 Coudry 2016.

9 Meier 1982, 442 : "After his return [de l'expédition en Afrique, donc après Thapsus], Caesar made conciliatory speeches to the Senate and people. They should have no anxieties: he did not wish to set up a tyranny, but to consult with the senators. He did in fact consult with them, but not very often". Nous n'avons eu accès qu'à la traduction anglaise de cet ouvrage rédigé en allemand. Peutêtre l'ouvrage original contient-il plus d'informations pouvant étayer cette représentation.

10 On trouve des discours, rapportés au style indirect, de César au Sénat chez César (B.C., 1.32 et 3.73). Le même procédé est utilisé à de très nombreuses reprises pour des contiones.

11 Les harangues rapportées sont en revanche bien plus nombreuses ; l'une des plus connues est le discours de César à ses soldats mutinés à Plaisance en 49 a.C. , qui se trouve chez Appien (B.C., 2.47) et Cassius Dion (41.27-35). On trouve chez Appien un discours à ses troupes à Brindes en 48 a.C. (B.C., 2.53 ; chez César : B.C., 3.6-7), et l'évocation du célèbre discours sur le Champ de Mars en 47 a.C., dans lequel César aurait appelé ses soldats quirites (2.93). Cassius Dion, qui évoque lui aussi ce discours et cette apostrophe $(42,53)$, consacre également, lors de la guerre des gaule, une harangue césarienne à ses soldats à Vesontio (38.34-47), analysée par Kemezis 2016. Sur la mutinerie de 47 a.C., voir Chrissanthos 2001.

12 Sall., Cat., 51. Ce discours ample et mesuré est construit selon les règles habituelles de la rhétorique classique.

13 Plut., Cat. Mi., 22.5.

14 Plut., Caes., 7.7-9.

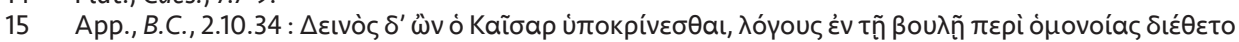

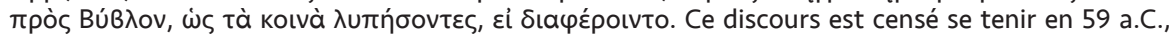
date à laquelle César partage le consulat avec Bibulus.

16 C.D. $41.15 .2-16.1$

17 La bibliographie est également en trompe-l'œil : de très nombreuses études évoquent César et la rhétorique, mais la plupart traitent, dans une optique historico-littéraire, des discours de César chez César ou de leur style, ou bien, dans une optique historique, de la formation rhétorique de 
Ce discours unique dans nos sources nous conduit à interroger en quoi la représentation de César en orateur dit quelque chose de la conception de Cassius Dion relativement à César et son pouvoir. Cela nous amènera à adopter une optique différente de celle de R. Étienne, car il ne s'agira pas de considérer d'emblée ce discours comme révélateur d'une conception politique générale de Dion ; il s'agira plutôt d'interroger la représentation qui nous est faite de César en orateur politique par Dion pour mettre en évidence quelle conception se fait Dion du pouvoir césarien. Pour ce faire, il sera nécessaire d'analyser non seulement le style de ce discours et ses articulations argumentatives, mais aussi la manière dont Dion l'insère dans son récit des événements de l'année 46 a.C. et l'inscrit en écho - ou non - à celui dont il est fait brièvement allusion au livre 41 .

\section{UN DISCOURS À PART}

Ce discours s'étend sur quatre chapitres (43.15-18). Il est possible qu'il ait été un peu plus long, mais s'il l'était, c'était vraisemblablement de quelques mots ou de quelques phrases seulement : une lacune est en effet relevée par le philologue H.S. Reimar dans son édition de Cassius Dion ${ }^{18}$ et sera ensuite signalée par les éditions suivantes jusqu'à l'editio maior de U. P. Boissevain ${ }^{19}$, qui estime que cette lacune était vraisemblablement assez circonscrite. Mais même avec quelques mots ou quelques phrases de plus, ce discours n'en aurait pas moins été le plus court de tous les discours que l'on trouve dans l'Histoire romaine, et ce de manière très nette : au livre suivant, le discours de Cicéron au Sénat mobilise onze chapitres (44.23-33) et celui d'Antoine lors des funérailles de César, quatorze (44.36-49). Le fameux débat entre Agrippa et Mécène mobilise douze chapitres pour l'un (52.2-13) et vingt-sept pour l'autre (52.1440). Le discours le plus long de l'Histoire romaine est celui de Cicéron au Sénat contre Antoine en 44 a.C. : il occupe trente chapitres (45.18-47). Après celui de César au Sénat au lendemain de Thapsus, les deux discours les plus brefs sont les harangues d'Antoine et d'Octavien à la veille d'Actium en 31 a.C., qui se déploient chacune sur sept chapitres (50.16-22 et 24-30).

Le César orateur représenté par Dion est donc un orateur fort peu prolixe. C'est déjà un élément invitant à la réflexion : pourquoi un personnage aussi important que César parle si peu au Sénat et devant le peuple ensuite ? Certes, il y a d'autres discours de César dans l'Histoire romaine : onze chapitres de harangue à ses officiers à Vesontio pendant la campagne contre Arioviste en 58 a.C. (38.36-46), et neuf chapitres de discours aux mutins de Plaisance pendant la guerre civile en 49 a.C. $(41.27-35)^{20}$. Mais, encore une fois, il s'agit de contiones. En revanche, au Sénat, César est représenté comme un orateur extrêmement succinct. Est-ce à dire que son discours est construit selon une esthétique de la breuitas, et que César est un orateur qui parle peu pour parler bien, ou, au contraire, est-ce là la marque d'un orateur de second plan, ce qui irait à l'encontre de la quasi-totalité des sources, qui qualifient César d'excellent orateur ?

Avant d'analyser plus précisément la construction de ce discours pour répondre à cette question, la comparaison avec le personnage qui parle le plus longuement dans les livres tardorépublicains de l'Histoire romaine conservés dans la traditions directe amène déjà un éclairage, car il s'agit de Cicéron : il cumule quarante-et-un chapitres de discours au Sénat ${ }^{21}$, sans compter

César. Voir notamment Rambaud 1987, Dangel 1995, Fantham 2009, Kraus 2010, Kemezis 2016 et Grillo 2017.

18 Reimar 1750-1752.

19 Boissevain 1895-1901.

20 Concernant les discours dans l'Histoire romaine, voir notamment Bellissime 2016a, Kemezis 2016, Mastrorosa 2017 et Burden-Strevens 2018.

21 Onze chapitres pour son discours au Sénat à la suite de l'assassinat de César en (44.23-33) et trente pour son discours au Sénat contre Antoine (45.18-47), les deux en 44 a.C. 
le dialogue Cicéron-Philiscos, qui court sur douze chapitres ${ }^{22}$. Cicéron, qui incarne la figure même de l'orateur, est un orateur prolixe. Il semblerait donc à première vue que Cassius Dion, en cantonnant la grande majorité des discours de César à des contiones, ait pour dessein de représenter César comme un chef de guerre brillant, mais comme un orateur de second rang. Si l'on compare ensuite la répartition de la parole de César avec celle d'Octavien-Auguste, qui est le personnage qui parle le plus longuement dans les livres impériaux conservés par la tradition directe, l'on constate qu'il incarne à la fois la figure du chef de guerre dans les livres tardo-républicains ${ }^{23}$ et de l'orateur ensuite ${ }^{24}$. Il est possible que cela soit révélateur : à première vue, Cassius Dion semble représenter un personnage qui, contrairement à César, a une parole performative tant lors des guerres civiles que dans la paix permise par sa victoire.

Dans son discours, César tente de rassurer quant à l'exercice du pouvoir qui sera le sien à présent qu'il est le vainqueur des guerres civiles. Pour ce faire, il décrit d'abord négativement quel ne sera pas son comportement, en mobilisant immédiatement les contre-exemples particulièrement éloquents de Marius, Cinna et Sylla ${ }^{25}(43.15 .3-4)^{26}$ :

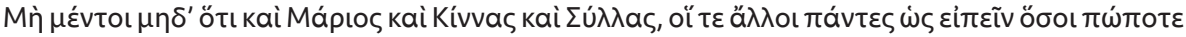

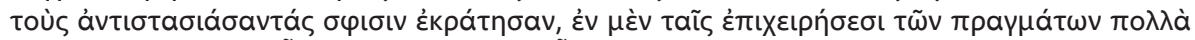

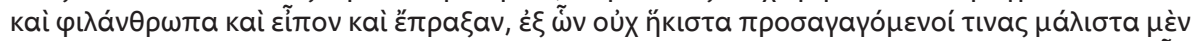

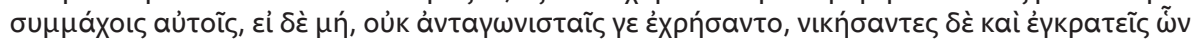

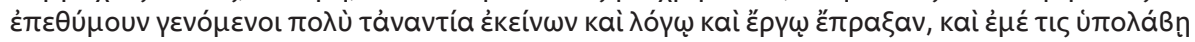

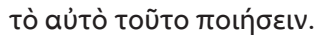

"Certes, Marius, Cinna, Sylla, et pour ainsi dire tous ceux qui ont jamais triomphé de leurs adversaires lors de guerres civiles, ont dit et fait beaucoup de bonnes choses au début de leur carrière, ce qui leur a valu d'attirer à eux un nombre considérable de gens, dont ils ont obtenu généralement le soutien actif, ou, à défaut, une absence d'hostilité mais, une fois vainqueurs et maîtres de ce à quoi ils avaient aspiré, ils se sont comportés de manière diamétralement opposée, en paroles comme en actes. Mais moi, que personne n'aille imaginer que je vais faire de même !"

Il récuse à la fois toute dissimulatio et toute aspiration à la tyrannie (43.15.5) :

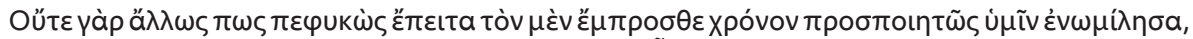

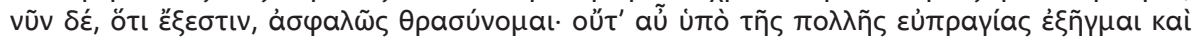

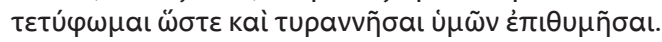

C.D. $38.18-29$

23 Il adresse une harangue à ses soldats à la veille d'Actium en 31 a.C. (50.16-22) : voir Mastrorosa 2014.

24 Voici la liste de ses discours : discours d'Octavien au Sénat de janvier 27 a.C. (53.3-10), discours d'Auguste au Sénat au moment de la loi Pappia-Poppaea en 9 p.C. (56.2-9), sans compter le dialogue Auguste-Livie en réaction à la conjuration de Cinna en 5 p.C. (55.14-21). Sur le plan rhétorique, Auguste semble faire miroir à Cicéron, bien que ses discours soient plus brefs que ceux de ce dernier: Cassius Dion compose deux discours au Sénat et un dialogue avec un interlocuteur pour ces deux personnages.

25 Les grands imperatores de la période républicaine sont régulièrement repris pour leur aspect paradigmatique dans les discours que l'on trouve dans l'Histoire romaine; aussi Agrippa et Mécène les évoquent-ils $(52.13 .2 ; 52.17 .3)$. Tibère, dans l'oraison funèbre qu'il prononce pour Auguste, réutilise ces exempla pour montrer qu'Auguste les a surpassés. Ces imperatores deviennent des exempla utilisés et réutilisés selon les besoins du discours. Voir Bellissime 2013. Il n'est donc pas étonnant qu'ici, César les utilise pour donner du crédit à son propos.

26 Récemment, C. Burden-Strevens a montré, pour ce discours de César chez Dion et pour ce passage en particulier, que César, au contraire de ses intentions affichées, faisait preuve d'une grande duplicité (Burden-Strevens 2020, 174-177 et 288-290). Il qualifie même ce discours de "'tyrant-speech' to the Senate" (p. 289). 
"En effet, il n'est pas vrai que dans mes relations avec vous, jusqu'à présent, j'aie dissimulé ma véritable nature, et que maintenant, parce que c'est possible et sans danger pour moi, je m'y laisse aller ; il n'est pas vrai non plus que mes nombreux succès aient provoqué chez moi une exaltation et un aveuglement tels que je désire vous gouverner en tyran."

Il s'appuie ensuite (43.16) sur des considérations générales sur le pouvoir et la gloire pour rassurer son auditoire ${ }^{27}$. Puis (43.17), positivement, il promet d'exercer le pouvoir comme un

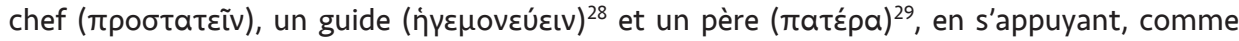
gage de sa bonne foi, sur son attitude clémente envers ses opposants lors des guerres civiles ${ }^{30}$, avant de présenter plus concrètement quels seront les fondements de ce pouvoir : des soldats - qu'il ne faut pas craindre - et des impôts un peu plus élevés que d'ordinaire pour pouvoir garantir la subsistance des soldats ${ }^{31}$ et embellir la ville (43.18.1) :

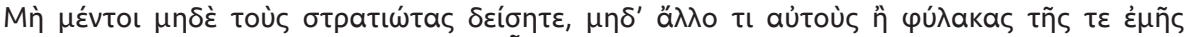

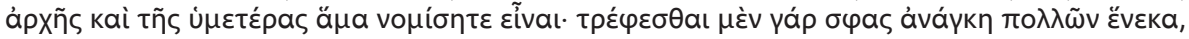

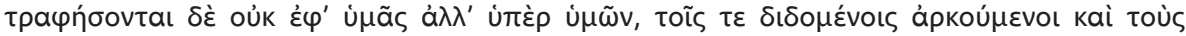

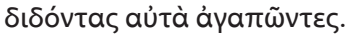

"Ne craignez rien, pas même mes soldats ! Et n'allez pas penser qu'ils sont autre chose que des gardiens de mon pouvoir tout autant que du vôtre. Il est nécessaire de subvenir à leurs besoins pour bien des raisons, mais l'on subviendra à leurs besoins non contre vous mais dans votre intérêt, car non seulement ils se satisferont de ce qu'on leur donnera, mais en outre ils seront reconnaissants envers ceux qui le leur auront donné."

Et un peu plus loin (43.18.2-4) :

27 Par exemple, C.D. 43.16.2 : "Car qui, plus que celui qui détient le pouvoir le plus élevé, se doit d'apporter les bienfaits les plus nombreux et les plus grands? Qui, plus que celui qui détient la plus grande puissance, se doit de commettre le moins d'erreurs? Qui se doit d'user des dons accordés par les dieux avec plus de prudence, sinon celui qui d'eux en a reçu les plus grands ? Qui se doit d'administrer les biens présents de la manière la plus droite, sinon celui qui en possède le plus grand nombre et qui craint le plus de les perdre ?"

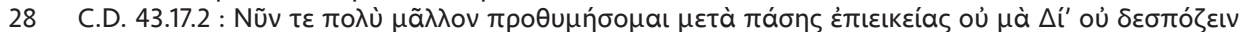

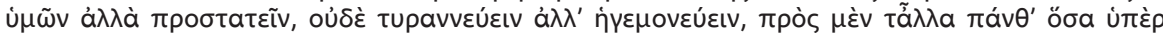

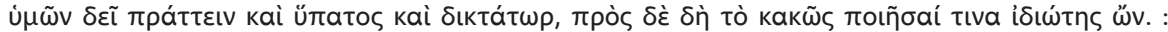
"Et désormais j'aurai bien plus à cœur, comme la convenance l'exige parfaitement, non pas - par Jupiter ! - d'être votre maître, mais votre guide, et non pas d'agir en tyran, mais en chef, puisque, pour absolument tous les actes qu'il faut accomplir en votre faveur, je serai consul et dictateur, mais simple particulier si je fais du tort à quelqu'un".

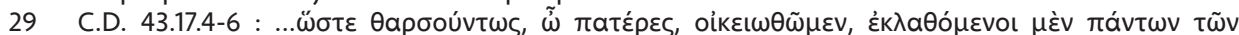

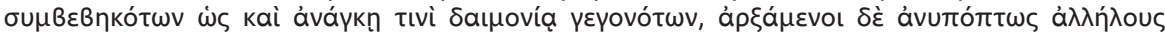

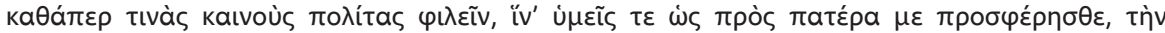

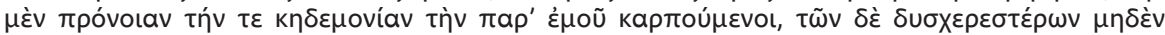

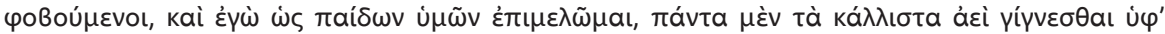

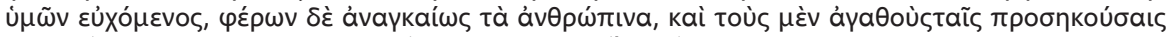

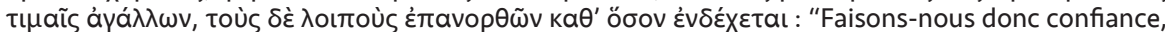
Sénateurs, dans nos relations, en effaçant d'abord le souvenir de tous ces événements passés survenus comme sous le coup de quelque divinité, et en commençant ensuite à avoir des liens d'amitié les uns pour les autres, sans se soupçonner, comme si nous étions de nouveaux citoyens, afin que de votre côté vous vous conduisiez envers moi comme à l'égard d'un père, profitant de ma prévoyance et de ma sollicitude, tout en n'ayant rien de plus déplaisant à redouter, et que de mon côté je prenne soin de vous comme de mes enfants, en priant que vous ne soyez les instigateurs que de ce qu'il y a de plus beau, tout en supportant toutefois les nécessités de la nature humaine, c'està-dire en décorant les bons citoyens d'honneurs correspondant à leur nature, mais en remettant les autres sur le droit chemin autant qu'il est possible."

30 C.D. $43.17 .3-4$

31 Le texte de Dion est cohérent car troupes et argent sont déjà présentés comme les fondements du pouvoir de César (C.D. 42.49.4). Ces deux fondements seront aussi ceux du pouvoir d'Auguste (C.D. 53.16.1) 
$\Delta$ เà yà

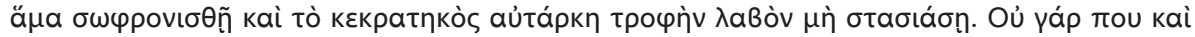

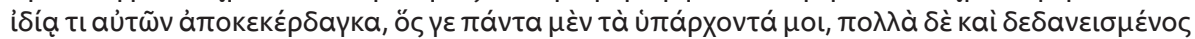

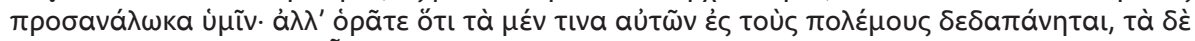

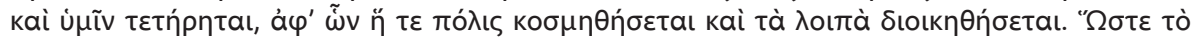

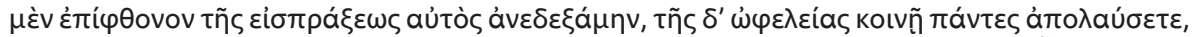

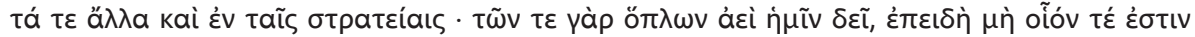

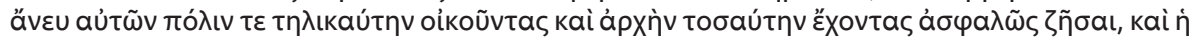

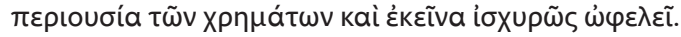

"C'est en effet pour cette raison que les sommes d'argent exigées sont plus élevées que d'ordinaire : cela permet à la fois que l'élément qui avait été séditieux s'apaise et que l'élément victorieux, puisqu'il reçoit une subsistance suffisante, ne devienne pas séditieux. Car bien entendu, je n'ai pas tiré profit de cet argent sur le plan privé, moi justement qui ai dépensé pour vous tout ce que j'avais et qui ai même emprunté beaucoup d'argent. Mais comprenez bien qu'une partie de cet argent a été utilisée contre les ennemis, tandis que l'autre a été conservée pour vous et permettra d'embellir la ville et d'administrer le reste des affaires. Ainsi ai-je pris sur moi l'aspect odieux du prélèvement d'impôt, tandis que vous tirerez collectivement jouissance de l'avantage qu'il suscitera, et tout particulièrement dans les campagnes militaires. Le fait est que nous avons toujours besoin des armes, puisque sans elles il ne nous est pas possible, à nous qui vivons dans une si grande cité et qui détenons un si grand empire, de vivre en sécurité ; et dans ce domaine aussi l'abondance de richesses est une aide puissante"

César conclut avec la promesse d'un prélèvement normal - c'est-à-dire sans instauration de nouveaux impôts ni exactions ${ }^{32}$.

Le César de Dion présente dans ce discours l'idéologie du pouvoir césarien selon Dion : il y affirme qu'il exercera certes le pouvoir seul, mais de manière modérée et pour le bien de tous. Ce pouvoir repose sur une adaptation assumée des institutions - César se pose en consul et en dictateur à la fois, ce qui est déjà le cas : la nouveauté semble résider dans l'annonce de la permanence de cette pratique institutionnelle. Mais cette adaptation est présentée comme positive : grâce à elle, César pourra mieux agir en faveur des Romains. Ce pouvoir n'a toutefois pas vocation à être excessif : César reste un simple particulier si jamais quelqu'un estime avoir été lésé ${ }^{33}$, garantissant ainsi la libertas et affichant le net refus de recourir à des

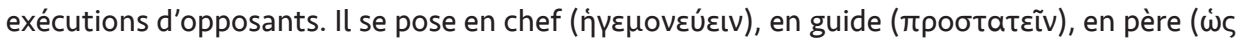
$\pi \rho o ̀ \varsigma ~ \pi \alpha \tau \varepsilon \dot{p} \rho)$ : il s'agit bien d'une relation asymétrique; pour autant, cette asymétrie se veut modérée et bienveillante, car César prétend refuser les aspirations à un pouvoir tyrannique

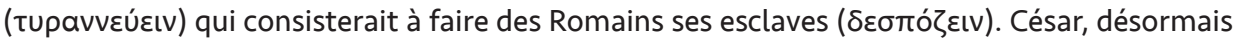

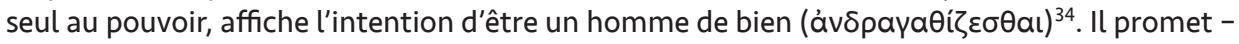
revendique même - sur le ton de l'aphorisme, un certain idéal de mesure et de sagesse pratique,

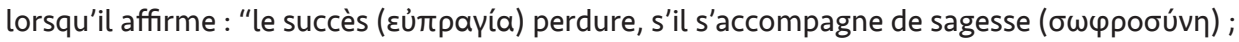

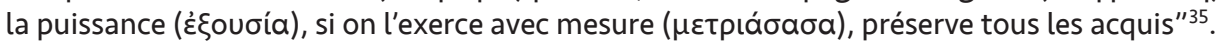

R. Étienne se fonde sur ce discours pour dire que Dion exprime à travers César sa conception de la monarchie idéale. En effet, il considère que l'œuvre du sénateur Cassius Dion est "marquée d'un égoïsme de classe, mis au service de sa communauté et au service de

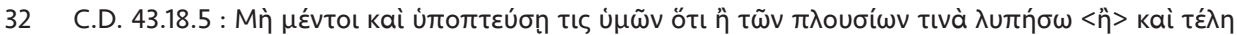

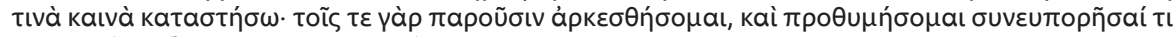

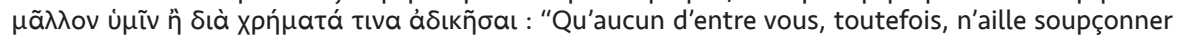
que je vais causer du tort à tel ou tel homme riche ou instituer de nouveaux impôts : je me contenterai des taxes présentes en ayant à cœur de vous aider de mes propres ressources plutôt que de traiter quelqu'un injustement à cause de son argent."

33 C.D. 43.17 .2

34 C.D. 43.15 .7

35 C.D. 43.16 .3$. 
l'empereur", et voit par conséquent dans ce discours la volonté de Dion de nous présenter "un César philosénatorial" et "l'image d'un régime monarchique fondé sur la vertu et la justice". "Ainsi Cassius Dion", poursuit-il, "parti d'un jugement politique catégorique et défendant le régime monarchique, nous présente un César qui se définit dans une discours capable de répondre à cet idéal politique". Il conclut en disant que le César de Dion est un "modèle d'idéal monarchique que les flatteries ont perdu" ${ }^{36}$.

C'est selon nous prendre le contenu du discours sans tenir suffisamment compte de sa composition, de son style et de son aspect performatif. Pour le dire plus simplement, l'analyse de R. Étienne voit dans ce César un théoricien du pouvoir et passe partiellement à côté de l'orateur qui nous est représenté. Nous n'avons nullement la prétention d'être exhaustif relativement à ce discours : aussi nous bornerons-nous à deux remarques.

1. Il semble tout d'abord relativement maladroit, pour quelqu'un qui essaie de rassurer un auditoire craignant des dérives tyranniques, de tenir un discours composé de cette manière. En effet, le discours s'ouvre en rappelant les excès de pouvoir de Marius, Cinna et Sylla, alors précisément que ce sont des excès de ce type qui suscitent l'inquiétude : évoquer d'emblée ce passé qui ne passe pas constitue un pari téméraire - et potentiellement à double tranchant - pour l'orateur, qui choisit certes de s'attaquer in medias res à la comparaison qui pourrait être faite entre lui et les précédents vainqueurs des guerres civiles afin de la désamorcer d'entrée de jeu, mais qui peut aussi bien braquer son auditoire en réactivant justement cette comparaison ${ }^{37}$. Cette maladresse se renforce quand César évoque la présence importante des soldats et un effort fiscal plus élevé que d'ordinaire : traiter ces deux aspects à une place aussi stratégique que la fin du discours apparaît très mal choisi devant un auditoire composé de partisans mais aussi d'anciens opposants dans la crainte d'une dérive du pouvoir de César.

2. Cette composition maladroite, ensuite, révèle de fait une structure souple et spontanée, assez loin des règles habituelles de la rhétorique : le discours, nous le disions quelques lignes plus haut, débute in medias res et se termine assez sèchement. On peut proposer deux interprétations à cela : soit l'on considère que l'exorde et sa traditionnelle captatio beneuolentiae sont absents, la péroraison manquant également, soit l'on peut au contraire concevoir que ces parties attendues sont à un endroit inattendu ; le passage 43.17.1 $1^{38}$, sorte de déclaration de sincérité, a une tonalité qui aurait pu être en adéquation avec un exorde, et le chapitre 43.16, dont on a parlé plus haut, avec son propos général et son accumulation de questions oratoires, aurait pu davantage correspondre à une péroraison : quoi qu'il en soit, en lieu et place de celle-ci, nous trouvons une évocation des impôts et des soldats de César, deux sujets d'inquiétude pour ceux qui l'écoutent. Que l'on suive l'une ou l'autre de ces hypothèses, il semble y avoir un écart de l'orateur par rapport à la structure

36 Étienne 1997, ibid.

37 Cet élément est également relevé par Burden-Strevens 2020, 288-289

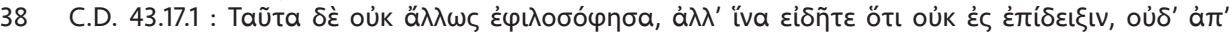

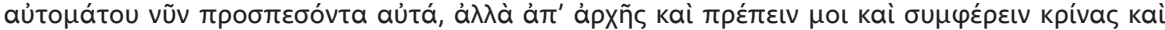

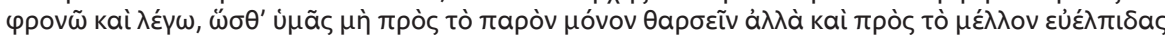

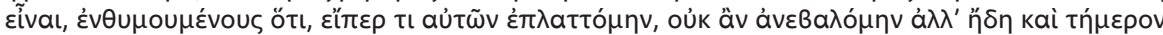

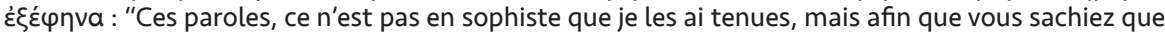
ces mots, ce n'est pas pour la montre, ni par hasard qu'ils se sont trouvés dans mon discours, mais parce que je les pense et que je les dis après avoir jugé que dès le début ils étaient convenables et utiles pour moi ; aussi pouvez-vous non seulement prendre courage pour le présent, mais aussi être confiants dans l'avenir, en considérant que si j'avais menti sur un point, je n'aurais pas attendu davantage et j'aurais déjà tombé le masque aujourd'hui à l'heure qu'il est." On trouve chez Dion (53.4) une semblable déclaration de sincérité dans la bouche d'Auguste en 27 lorsqu'il feint de remettre tous ses pouvoirs; voir Bellissime \& Hurlet 2018, 5 et 47-49. 
attendue. Cet écart fait de César un orateur particulier, voire paradoxal, en ce qu'il refuse de se plier aux règles de la rhétorique.

Pour tenter de comprendre le sens de cet écart, la comparaison avec les deux orateurs du livre suivant est éclairante. Cicéron fait un discours dans un contexte d'urgence ${ }^{39}$-César vient d'être assassiné - qui invite à être bref et pragmatique dans ses propos ; aussi la structure est-elle relativement sobre, certes, mais un soin est tout de même porté dans les parties stratégiques du discours, notamment au début : Cicéron affirme, dans une rupture de construction qui recentre son propos, qu'il "ne veut pas commencer son discours par des propos désagréables" 40 ; César, en revanche, commence son discours en évoquant, on l'a vu, le souvenir de Marius, Cinna et Sylla. Le Cicéron que donne à voir Dion semble donc prendre soin, même dans un discours sobre, de choisir ses mots prudemment. Quant à Antoine, il fait une oraison funèbre lors des funérailles de César ; c'est un discours très intéressant car Cassius

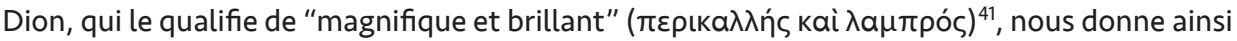
à voir ce qu'il considère comme un discours très réussi. Or ce discours à la gloire de César est relativement ample et suit assez traditionnellement la composition rhétorique traditionnelle, avec un exorde comprenant une captatio beneuolentiae (44.35), puis le récit de la vie de César selon le canon de l'oraison funèbre ${ }^{42}$, et une péroraison des plus flamboyantes (44.49). On le voit, ce discours s'oppose radicalement à celui de César, qui est bref et à la composition assez lâche. Pour Dion, le discours d'Antoine n'est pas nécessairement idéal, mais il est beau ; Dion semble donc, avec l'orateur César, construire un discours qui au contraire n'est pas esthétiquement de haut niveau.

Cette première lecture du discours de César au Sénat au lendemain de Thapsus accentue encore le caractère hors-norme de ce discours unique. Dion semble délibérément faire tenir à César un discours relativement maladroit dans sa construction et assez peu esthétique, ce qui confirme notre hypothèse de départ, fruit d'une brève comparaison entre César et les autres orateurs principaux de l'Histoire romaine : César semble ici dépeint comme un orateur plutôt médiocre.

\section{UN DISCOURS PEU CONVAINCANT}

Comment expliquer qu'un personnage de l'importance de César soit ainsi représenté ? Nous allons tenter d'y répondre en effectuant une deuxième lecture de ce discours tenant compte de son insertion dans le récit historique.

Le discours de César au Sénat après Thapsus s'inscrit chez Dion dans une séquence politique plus large (C.D. 43.14-27) que l'on peut subdiviser en trois mouvements : d'abord, le vote par le Sénat, avant le retour de César d'Afrique, de décrets en l'honneur du dictateur (C.D. 43.14$)^{43}$; puis, celui-ci rentré, de la célébration de son quadruple triomphe - sur la Gaule,

39 C.D. $44.23-33$.

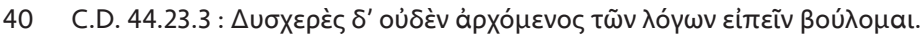

41 C.D. 44.35 .4

42 Sont évoqués d'abord sa personnalité en tant qu'individu, en faisant état de sa famille (C.D. 44.37), de son éducation (C.D. 44.38) et de son caractère (C.D. 44.39), puis, après une transition (C.D. 44.40), sa carrière politique : sa préture en Espagne (C.D. 44.41), ses actions à Rome et ses conquêtes (C.D. 44.42-43), son rôle dans les guerres civiles (C.D. 44.44-45), l'enrichissement de Rome qu'il permit, notamment grâce à l'acquisition de l'Égypte (C.D. 44.46), son rôle de pacificateur au sortir des guerres civiles (C.D. 44.47). Avant la péroraison (C.D. 44.49), un rappel des titres de César est effectué (C.D. 44.48). Sur l'éloge funèbre, voir Kierdorf 1980, et sur celle en particulier d'Antoine aux funérailles de César, voir Pepe 2011.

43 Le Sénat, selon Dion, a décrété quarante jours de supplications d'actions de grâces pour célébrer sa victoire, le droit de triompher sur un char tiré par des chevaux blanc et d'être alors escorté par 
l'Égypte, le Pont-Euxin et l'Afrique -, à l'occasion duquel furent donnés d'importants jeux du cirque (C.D. 43.19-24) ; enfin, les réformes de César (C.D. 43.25-27). Le discours que nous analysons se trouve donc après le vote des honneurs à César et avant la célébration par celui-ci de son triomphe. Voici précisément comment Dion justifie l'insertion de ce discours dans son récit (C.D. 43.15.1-2) :

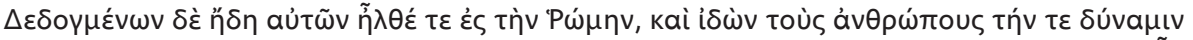

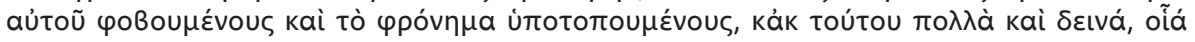

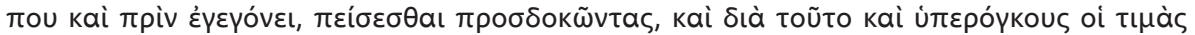

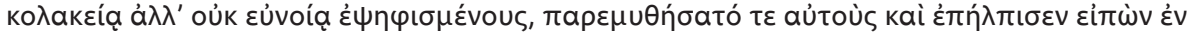

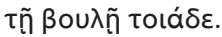

"Ces décrets étaient déjà votés à l'arrivée de César à Rome. Quand il vit que les gens craignaient son pouvoir et se méfiaient de ses intentions, qu'ils s'attendaient par conséquent à subir de nombreux malheurs, comme ceux, je suppose, qui s'étaient produits naguère et que c'était pour cette raison qu'ils lui avaient voté, par flagornerie et non par bienveillance, des honneurs excessifs, il chercha à leur rendre confiance et espoir en prononçant au Sénat le discours suivant."

On le voit, le discours de César est motivé par un double constat : les gens craignent son

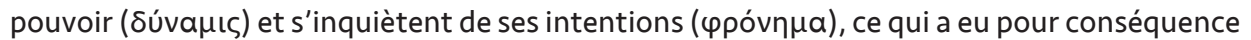

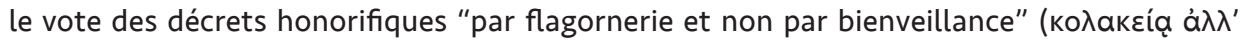
oủk عủvoíą).

Pour Dion, c'est donc l'inquiétude qui motive ces honneurs. Le vocabulaire employé est troublant, car dans son discours, César utilise des mots semblables lorsqu'il tente de rassurer les sénateurs (C.D. 43.16.3-4) :

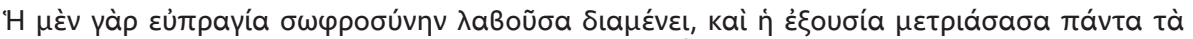

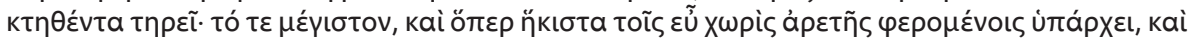

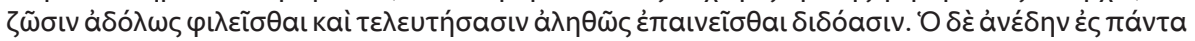

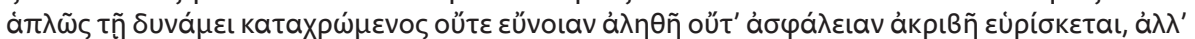

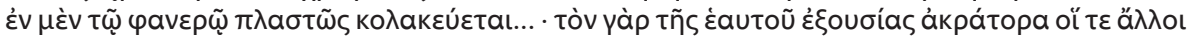

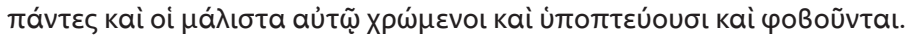

"Car le succès perdure, s'il s'accompagne de sagesse, et le pouvoir, s'il s'exerce avec mesure, préserve tous les acquis, et - c'est là l'élément le plus important et qui fait le plus défaut à ceux qui réussissent sans user de vertu - ces comportements valent à ceux qui les adoptent d'être réellement aimés durant leur vie et de recevoir après leur mort des éloges véridiques, alors que celui qui use de son pouvoir sans restriction et dans toutes les situations, ne trouve jamais ni bienveillance véridique ni sécurité authentique, mais reçoit en public de feintes flagorneries, car celui qui ne contrôle pas son propre pouvoir, tous - et surtout ceux qui le fréquentent le plus éprouvent à son encontre méfiance et crainte".

On peut raisonnablement penser que le choix du lexique n'est pas laissé au hasard. La description que fait César du mauvais monarque, qui "use de son pouvoir sans restriction et

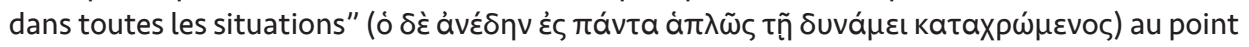

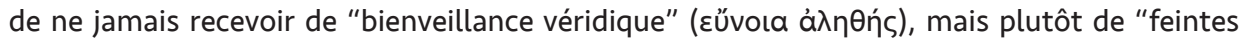

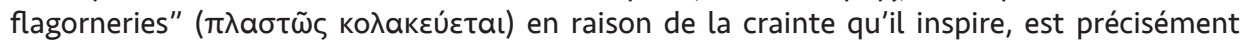

une foule de licteurs, le titre de préfet aux moeurs pour trois ans, la dictature pour dix ans, le droit de s'asseoir au Sénat sur la chaise curule aux côtés de consuls en charge et de toujours donner son avis en premier, le droit de donner le signal lors des courses de chars, le droit de nomination des magistratures attribuées jusqu'ici par le peuple, l'érection d'un groupe statuaire à son image sur le Capitole avec la dédicace "au dieu invincible" et l'inscription de son nom à la place de celui de Catulus sur le temple de Jupiter Capitolin. 
l'image, selon Dion, qu'a César aux yeux des Romains à ce moment précis. Tout se passe comme si par ce procédé Cassius Dion nous donnait à entrevoir par anticipation l'échec politique de César, d'autant plus que ce passage est également teinté d'une forte ironie tragique : celui qui

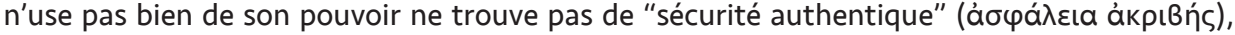
affirme César ; or César sera assassiné par des conjurés environ un an et demi plus tard, aux Ides de mars 44 a.C. Il faut d'ailleurs noter un chiasme dans cette description du mauvais

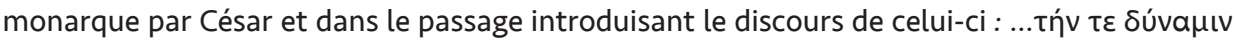

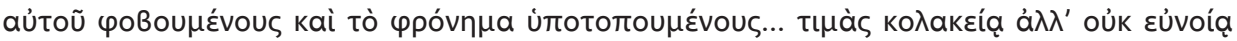

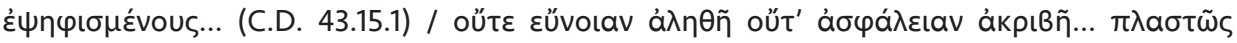

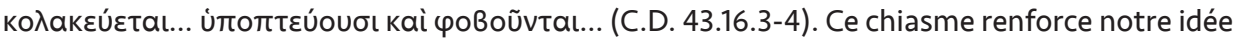
d'une stratégie d'écriture délibérée de la part de Dion et nous permet de penser que comme l'affirmait Boissevain, la lacune à cet endroit devait être très circonscrite ${ }^{44}$.

L'introduction au discours de César nous permet donc de mettre en évidence des stratégies d'écriture révélatrices de sa conception du pouvoir césarien en 46 a.C. : par une mise en abyme renforcée d'ironie tragique, Dion donne à voir, au lendemain de Thapsus, un César qui inquiète et qui, si son discours ne parvient pas à rassurer, risque de se faire renverser en raison de la crainte qu'il inspire. Or le lecteur sait que c'est précisément ce qui va arriver : Dion nous fait entrevoir par anticipation l'échec politique de César à travers son échec rhétorique.

En effet, le César orateur que représente ici Dion est en situation d'échec : on le voit dans sa manière de commenter les réactions à ce discours (C.D. 43.18.6):

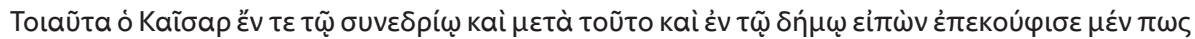

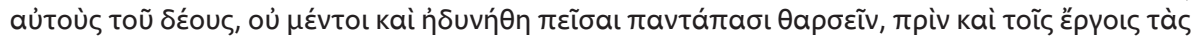

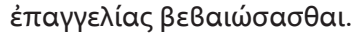

"Voilà quelles furent les paroles que César prononça au Sénat puis devant le peuple ; ainsi, il apaisa dans une certaine mesure les craintes, mais fut incapable de persuader de reprendre totalement confiance tant qu'il n'aurait pas confirmé ces annonces par des actes".

Le texte que nous analysons apparaît de plus en plus hors-norme : alors que les sources littéraires évoquent de manière unanime un César orateur exceptionnel, Dion montre ici, tant explicitement qu'implicitement, un discours qui échoue. En effet, Dion affirme explicitement que César a rassuré, mais "dans une certaine mesure" ( $\pi \omega \varsigma)$ seulement, ce qui revient à dire

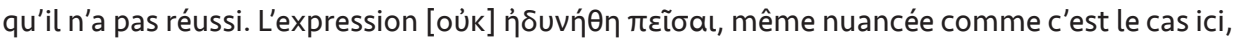
est loin d'être un compliment pour un orateur. Cassius Dion souligne d'ailleurs régulièrement le rôle actif du discours sur le cours de l'histoire : le discours de Cicéron au Sénat au lendemain des Ides de mars est immédiatement suivi d'effet ${ }^{45}$ - l'amnistie et la ratification des actes de César sont votées par le Sénat que l'orateur a convaincu -, celui d'Antoine aux funérailles de César met le feu aux poudres et réactive les guerres civiles ${ }^{46}$. Ces deux discours font d'autant plus ressortir l'échec de César ${ }^{47}$.

44 L'argumentation peut se comprendre sans avoir à faire l'hypothèse d'une lacune longue ; le yáp introduit la cause du fait que les mauvais monarques ne suscitent pas la bienveillance et ne sont pas en sécurité : c'est parce qu'on se méfie d'eux et qu'on les craint. On peut même faire l'hypothèse, à

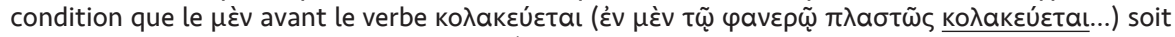
solitarium, chose rare mais pas impossible) qu'il n'y ait d'ailleurs aucune lacune. De surcroît, cela pourrait avoir du sens : l'absence de ce second membre de la parataxe qui semble faire défaut - et que le lecteur, qui l'attend, supplée de lui-même - peut renforcer l'ironie tragique du passage.

45 C.D. 44.34.1.

46 C.D. 44.50 .1$.

47 On peut ajouter un troisième éclairage : celui du discours d'Auguste en 27 (C.D. 53.3-10) qui réussit précisément là où César a échoué - des allusions à César sont d'ailleurs exprimées (C.D. 53.6.4 et 7.3). 


\section{UN ÉCHEC RHÉTORIQUE ET POLITIQUE DÉJÀ ANNONCÉ}

Cassius Dion, de plus, montre dans la suite de son récit que César n'a précisément pas mis en conformité ses actes et ses paroles, ce qui revient à insister implicitement sur l'échec de César, qui n'est pas en mesure de rassurer.

En effet, les chapitres qui suivent ce discours sont consacrés à la célébration du quadruple triomphe de César. Or il est fait mention, à plusieurs reprises et de manière plus ou moins explicite, de la démesure et de l'extravagance de ces célébrations, provoquant un malaise latent mais palpable. Dans la procession triomphale, les Romains apparaissent choqués pour trois raisons : par le fait que César célèbre une victoire, dans le cas de l'Afrique, contre d'autres Romains ; par la présence de la princesse égyptienne Arsinoé, qui apparaît enchaînée ; et par le nombre de licteurs entourant César ${ }^{48}$. Ce passage, qui suit immédiatement le discours de César, semble d'emblée montrer que les actes de César diffèrent des promesses apaisantes qui viennent d'être rapportées. La population, que Dion décrivait comme inquiète, l'est toujours autant ici : ni son discours ni son comportement n'ont donc rassuré qui que ce soit. À propos des jeux du cirque célébrés en cette occasion, Dion souligne ensuite de manière très claire l'extravagance dépensière ${ }^{49}$ de César, notamment lorsqu'il rapporte que, pour protéger les spectateurs du soleil, il fit tendre un voile de soie au-dessus de leurs têtes ${ }^{50}$. Ces excès dans la célébration des malheurs causés par les guerres civiles et dans les dépenses suscitent l'indignation, mais les civils ne peuvent exprimer ce mécontentement, vraisemblablement par crainte de répression ${ }^{51}$, alors que César avait pourtant promis de garantir la libertas. Non seulement ses paroles n'ont pas pu rassurer, mais encore ses actes sont en décalage avec ses paroles.

Le contexte immédiat de ce discours nous permet d'affirmer que Cassius Dion met en scène l'échec rhétorique de César et préfigure déjà les ides de mars 44. Mais cet échec rhétorique est en réalité déjà annoncé par l'autre discours de César au Sénat que rapporte Cassius Dion pour l'année 49 a.C. ${ }^{52}$; il en est d'une certaine façon le doublon narrativisé. En effet, c'est, comme après Thapsus en 46 a.C., l'inquiétude qu'il ressent à son égard - et notamment à cause de ses troupes nombreuses dans la Ville - qui motive sa prise de parole, en des termes très semblables (C.D. 41.15.2-3) :

48 C.D. 43.19 : "Ensuite, il organisa des festivités éclatantes, comme il convenait naturellement pour de si importantes et nombreuses victoires, et parmi ces festivités se distinguait tout particulièrement le quadruple triomphe qu'il célébra, lors de quatre journées différentes, sur les Gaulois et sur l'Égypte, sur Pharnace et sur Juba. Tout cela ne manqua pas de réjouir les spectateurs. En revanche, la vue de l'Égyptienne Arsinoé - César la fit défiler parmi les prisonniers de guerre -, de la foule des licteurs et des citoyens tombés en Afriques et montrés lors de la procession, furent pour eux un spectacle terriblement douloureux. En effet, les licteurs, du fait de leur nombre, formaient à leurs yeux une masse inquiétante car auparavant ils n'en avaient jamais vu autant en même temps ; et le spectacle d'Arsinoé enchaînée, une femme, naguère considérée comme une reine - chose qui, du moins à Rome, n'était jamais arrivée -, suscita une pitié unanime. Après quoi, c'est sous couvert de ce prétexte qu'ils se lamentaient aussi sur leurs propres malheurs. Si elle fut néanmoins relâchée grâce à ses frères, d'autres, dont notamment Vercingétorix, furent mis à mort".

49 À ce sujet, voir Newbold 1975. Cet article recense tous les passages consacrés aux jeux du cirque dans l'Histoire romaine et montre que, lorsqu'elle est explicitement exprimée, l'opinion de Cassius Dion sur les jeux et les dépenses qu'ils impliquent est presque toujours dépréciative.

50 C.D. 43.24.1-2: "Si je mentionne une seule anecdote, révélatrice de l'extravagance qui était la sienne à ce moment-là, je donnerai alors une idée de tout le reste. Pour qu'aucun des spectateurs ne soit gêné par le soleil, César fit tendre au-dessus d'eux des voiles qui - certains l'affirment - étaient en soie. Ce type de toile est une confection du luxe barbare, importé de chez eux jusqu'à chez nous aussi pour satisfaire l'extrême raffinement des femmes de la haute société".

51 C.D. 43.34.3.

52 Ce discours narrativisé intervient dans un contexte relativement similaire : en 49, César investit Rome, abandonnée des pompéiens, et César adresse ce discours pour rassurer quant à ses motivations. 


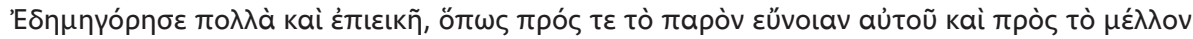

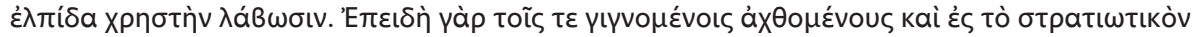

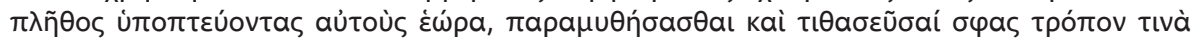

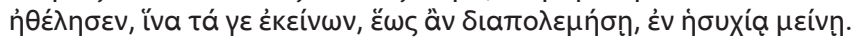

"Il fit un long discours, mesuré dans ses termes, pour amener les sénateurs à concevoir dans l'immédiat des dispositions favorables à son égard et, à plus long terme, de solides espoirs : comme il voyait qu'ils étaient fâchés de la tournure des événements et que la foule de ses soldats les rendait méfiants, il voulut trouver le moyen de les amadouer et de les apprivoiser, en quelque sorte, de façon que, de leur côté en tout cas, les choses fussent calmes jusqu'à ce qu'il terminât la guerre".

César à ce moment-là aussi cherche à apaiser et se refuse à employer des menaces contre ceux qui lui resteraient hostiles, ou qui seraient neutres ${ }^{53}$. Et de la même manière, le discours est ensuite dupliqué devant le peuple ${ }^{54}$, qui, déjà, craignait que César n'agisse comme un Marius ou un Sylla ${ }^{55}$. Après ce discours prononcé en 49 a.C., ses actes semblaient déjà ne pas être en adéquation avec ses annonces, comme le montre la manière dont, selon Dion, César a traité un tribun de la plèbe nommé Lucius Metellus et la manière dont il s'est emparé du Trésor ${ }^{56}$; Dion affirme que cet acte avait été fait "au nom de la démocratie" (óvó $\mu a \tau \iota \mu$ c̀v íoovouías)

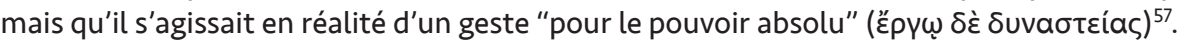

Ce discours narrativisé est toutefois différent sur certains points. César, en 49 a.C., tente d'acheter la paix et sa légitimité par une distribution de blé et par la promesse d'un don de soixante-quinze deniers à tous les citoyens - ce sera une promesse non tenue (C.D. 41.17.1) ${ }^{58}$ :

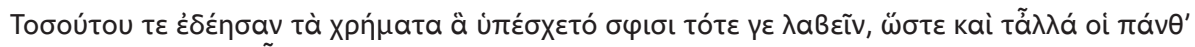

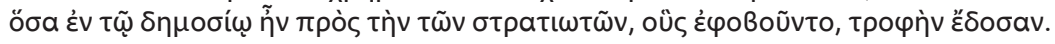

"Les citoyens furent si loin de toucher, à ce moment-là du moins, l'argent que César leur avait promis, qu'il leur fallut donner tout celui qu'il y avait notamment dans le trésor pour entretenir ces soldats qui les terrorisaient".

L'autre différence fondamentale entre ces deux discours de César au Sénat, c'est que celui de 49 a.C., s'il donne moins à voir des propos de César, laisse en revanche entrevoir bien davantage des réactions du peuple. Or ces réactions que rapporte Dion montrent que personne n'est dupe des promesses de César ni même rassuré, au contraire (C.D. 41.16.1-4) :

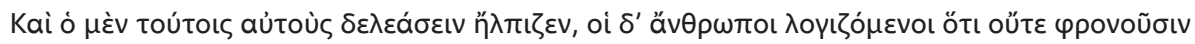

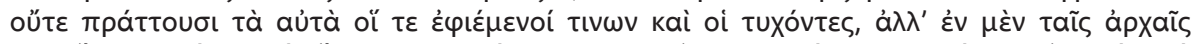

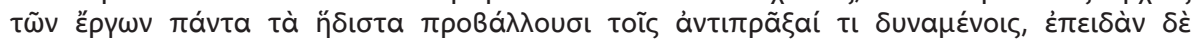

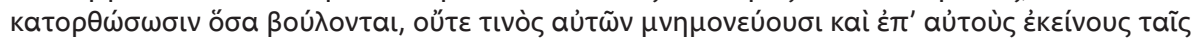

C.D. 41.15 .4 : "Voilà pourquoi il ne s'en prit à aucun d'entre eux ni ne prononça aucune menace ; au contraire, il fit une sortie à caractère imprécatoire contre ceux qui avaient choisi de faire la guerre à leurs concitoyens et, en guise de péroraison, proposa que, sans plus attendre, on envoyât des ambassadeurs aux consuls et à Pompée pour défendre la paix et la concorde entre eux".

54 C.D. 41.16.1.

55 C.D. 41.16 .3

56 C.D. 41.17.2.

57 Nous reprenons pour tous les passages du livre 41 la traduction de la CUF. Toutefois, les mots

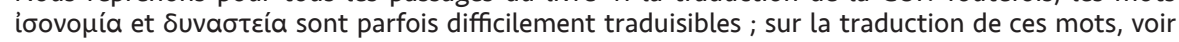
Cordier 2003, Coudry 2016 et Bellissime 2016a et 2016b.

58 Cette promesse, plus exactement, sera tenue avec du retard, qui se sera compensé par des intérêts, comme le montre 43.21.3 : "À la plèbe frumentaire, il distribua aussi les soixante-quinze deniers

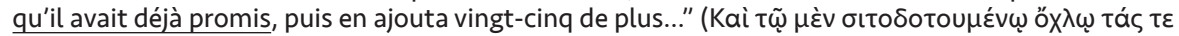

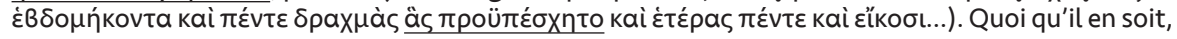
au moment où César s'exprime, cette promesse est pour lors non tenue. 


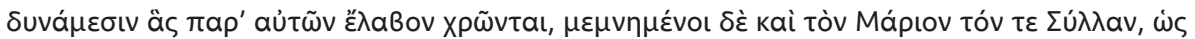

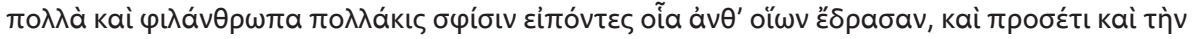

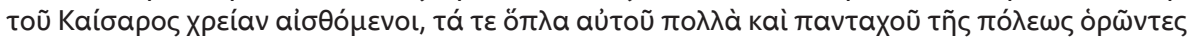

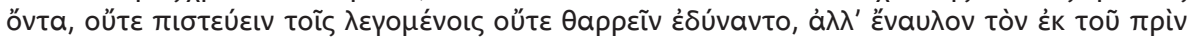

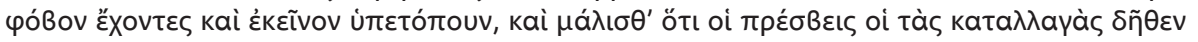

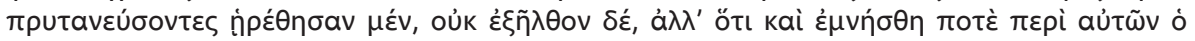

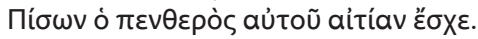

"S'il espérait les appâter ainsi, eux se disaient que les sentiments et les actions des gens sont différents selon qu'ils désirent une chose ou qu'ils l'ont obtenue et qu'au début d'une entreprise ils ne lésinent pas sur les promesses les plus alléchantes à qui pourrait leur faire obstacle, mais que lorsqu'ils ont atteint leurs objectifs ils oublient tout cela et tournent les instruments du pouvoir contre ceux-là même grâce à qui ils les ont en mains ; les citoyens se souvenaient de Marius et de Sylla, de ces nombreux discours bienveillants souvent répétés et de ce qu'en lieu et place ils avaient fait ; et puis pour César, on voyait bien où il voulait en venir et on constatait qu'il avait beaucoup d'armes, partout dans la ville, et cela ne pouvait pas donner confiance dans les paroles ni rassurer ; au contraire, on tremblait encore de ce qui venait de se passer et on avait des doutes sur lui, d'autant que les ambassadeurs qui devaient négocier tout de suite la réconciliation avaient été désignés, mais n'étaient pas partis et qu'au contraire lorsqu'un jour Pison, son beau-père le lui avait rappelé, César lui en avait fait grief".

En conséquence, au moment où César prend la parole au Sénat puis devant le peuple au lendemain de Thapsus, il apparaît déjà délégitimé en tant qu'orateur, puisqu'il a tenu quelques années plus tôt le même discours ou presque, contenant les mêmes promesses ou presque, et que ce discours et ces promesses, qui n'avaient déjà pas convaincu, n'avaient en outre pas été suivis d'effets. Au vu de la situation telle que la décrit Cassius Dion, le Sénat et le peuple n'ont donc absolument aucune raison d'être rassurés par ce discours de 46 a.C. De plus, César ne propose pas d'argent à ce moment, mais se contente d'exposer de manière très générale des considérations sur le pouvoir qui se veulent rassurantes, à côté de l'évocation de ses soldats et de l'effort fiscal à venir : ce discours, très vraisemblablement, est encore moins susceptible de convaincre ; à ce propos, Cassius Dion affirme d'ailleurs quelques chapitres auparavant que c'est parce que César a toujours pris soin de proposer des avantages matériels qu'il a réussi à convaincre certains soldats du camp opposé de le rejoindre, quand les partisans de Pompée se contentaient de belles paroles générales et de ce fait peu efficaces ${ }^{59}$.

\section{CONCLUSION}

Le discours de César au Sénat au lendemain de Thapsus, par une construction plutôt maladroite et une composition semble-t-il peu soignée, paraît donc fort peu susceptible de convaincre, pas plus qu'il ne semble capable de séduire par son esthétique; cela conduit à une représentation étrange d'un César à l'apogée de son pouvoir mais impuissant comme orateur. L'éclairage de son insertion dans la matière narrative et des échos à cet autre discours narrativisé du vainqueur de Pompée accentue encore ce sentiment : Dion semble mettre en scène l'éclosion paradoxale d'une parole inaudible. C'est pour cette raison que la lecture qu'en propose R. Étienne nous semble à compléter. Indépendamment de la conception politique que l'on prête au sénateur de l'époque sévérienne Cassius Dion, ce qui semble se jouer dans 
ce texte est une représentation du pouvoir césarien : l'historien donne à voir un César qui sort vainqueur des guerres civiles mais ne parvient pas, en définitive, à gagner la paix, car il échoue à désamorcer les inquiétudes - fondées ou non - des sénateurs et du peuple romain à son égard. C'est la raison pour laquelle Cassius Dion adopte un procédé d'écriture pour le moins étonnant : il compose un discours de César, au style direct, pour mettre en scène cet échec politique, celui de César orateur - bientôt celui de César dictateur.

\section{Antoine Jayat}

Université Bordeaux Montaigne UMR 5607 Ausonius

Retrouvez la version en ligne gratuite et ses contenus additionnels
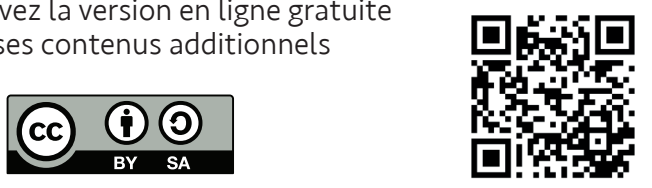